\title{
Shifting Cultivation in der Selva Lacandona (Mexiko)
}

Die Shifting Cultivation basiert auf einem natürlichen Rotationssystem, bei dem Landflecken durch Roden bzw. Brandroden gesäubert werden, mit einfachen Geräten gepflanzt und später von Hand geerntet wird. Das nach einigen Ernten verbrauchte und aufgegebene Feld regeneriert in einer natürlichen Umwelt relativ rasch.

Diese einfache Wirtschaftsweise ist auch heute noch, vor allem in den Tropen, weit verbreitet. So schätzte man 1968, daß noch mehr als 200 Millionen Bauern irgend eine Form der Shifting Cultivation auf 20\% des Festlandes oder $44 \%$ der landwirtschaftlich nutzbaren Fläche der Erde ausübten (MUENCH - NAVARRO 1978). Die Shifting Cultivation wird, zusammen mit Raubbau betreibenden Holzgesellschaften usw., für die ein alarmierendes Ausmaß erreichende Zerstörung des tropischen Regenwaldes verantwortlich gemacht. Allein für das tropische Amerika schätzt man die jährliche Zerstörungsrate des Waldes zwischen 1976-80 auf 4119000 ha (FAO 1981). Ziel der vorliegenden Arbeit war es, in der Selva Lacandona, einem mehr als $13000 \mathrm{~km}^{2}$ grossen, von vorwiegend hohem tropischen Regenwald bedeckten Gebiet im Süden Mexikos, die lokale Ausprägungsform der Shifting Cultivation (roza, tumba y quema genannt) und deren Zusammenhang mit den gravierenden Waldveränderungen zu untersuchen (Abb. 1). Dabei wurde die Untersuchung der Shifting Cultivation in der Form einer Fallstudie durchgeführt, weshalb die Angaben z. T. sehr detailliert sind.

\section{Shifting Cultivation}

In der Selva Lacandona wird in zwei Zyklen angebaut, die sich zeitlich überschneiden und zwei Ernten im Jahr ermöglichen. Der Hauptanbau wird umilpa de año» genannt, der zweite Zyklus «milpa de tornamil» (Abb. 5).

\subsection{Der Anbauzyklus «milpa de año»}

\subsubsection{Die Auswahl des Feldes}

Die Auswahl des zu bearbeitenden Stücks Landes erfolgt nach rationalen Gesichtspunkten. Grundsätzlich werden Ebenen steilen, unzulänglichen Gebieten vorgezogen, doch ist dies vielerorts wegen der sich

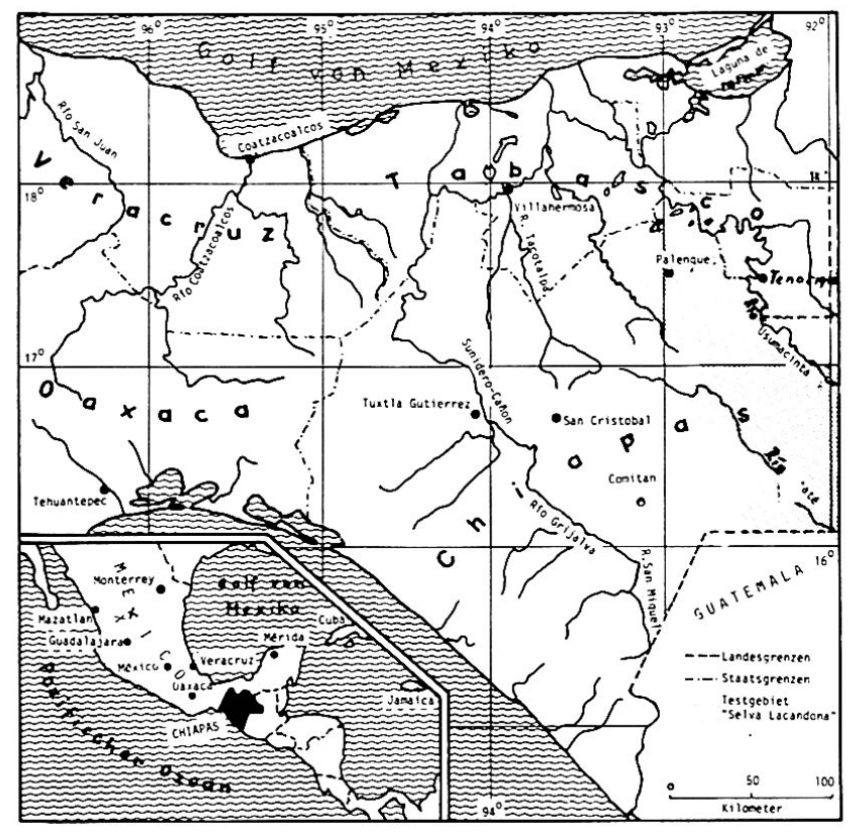

Abb. 1 Karte des Untersuchungsgebietes "Selva Lacandona".

ausbreitenden Viehzucht nicht mehr möglich. Eine Bearbeitung steiniger Hänge ist schwieriger und bringt kleinere Erträge. Älterer Sekundärwald (acahual) wird bevorzugt. Dies ist deshalb so, da zum Roden von Primärwald (monte) eine Erlaubnis notwendig ist (nur die Lacandonen-Indianer benötigen zum Roden von Primärwald keine Bewilligung). Diese Erlaubnis kann auf der «Secretaria Forestál y de la Fauna» für 300 bis 1000 Pesos erhalten werden. Das Fällen nutzbarer Bäume, wie Cedrillo (Guarea sp.), Caoba (Swietenia macrophylla) und Canshán (Terminalia amazonia), etc. ist nur der Forstbehörde gestattet. Das Fällen von Urwaldriesen des Primärwaldes ist zudem ungleich mühsamer und arbeitsaufwendiger. Bei älteren $a c a-$ huales brennen die Feuer bei der "quema" besser als bei Primärwald. Schließlich scheinen auch die AnbauErträge bei alten Sekundärwäldern besser zu sein als bei Primärwald (TUR Rent-Fernandez 1978, p. 115).

Stauende Nässe wirkt sich negativ auf die Kulturpflanzen aus. Feuchte Stellen werden also tunlichst

Michel Gelbert, dipl. Geograph,

Geographisches Institut der Universität Zürich,

Postfach, 8033 Zürich 


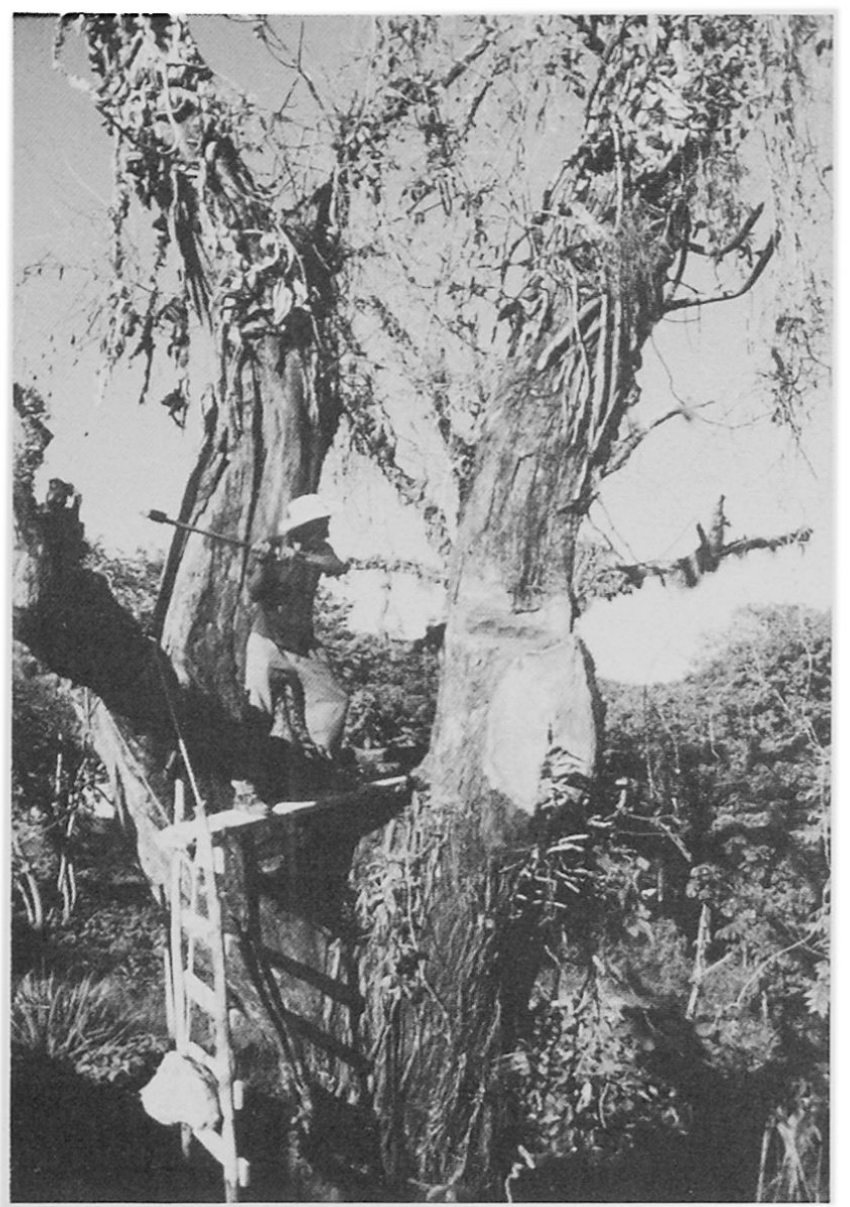

Abb. 2 Das Fällen dickstämmiger Bäume erfolgt von einer ca. $3 \mathrm{~m}$ hohen Plattform aus (bei Palenque, 27.11.81).

gemieden. Als Feuchtezeiger gelten z. B. die Caoba (Swietenia macrophylla) oder der Cedro (Cedrela odorata).

Trotzdem wurden immer wieder Felder angetroffen, die unter Wasser lagen. Der Boden muß möglichst tief sein und wenn möglich eine schwarze Farbe aufweisen. In Feldern mit gelben Böden oder mit roten Steinen (Eisen + Magnesium) werden schlechte Resultate erzielt. Die Bauern kennen also sehr wohl Kriterien zur Auswahl des neuen Feldes (nicht dieser Meinung ist z. B. WATTERS 1971, p. 122). Aber: Es ist von den Bauern zum voraus nicht zu erfahren, wohin sie im nächsten Zyklus zu ziehen gedenken.

Die Größe der Felder schwankt zwischen einigen Quadratmetern und 10 ha. Letzteres stellt aber eine absolute Ausnahme dar; es handelt sich um das gemeinsam bewirtschaftete Feld eines Ejidos (1). (Grundsätzlich lassen sich zwei Formen von Besitzverhältnissen in der Selva Lacandona unterscheiden: Als Ejido (1) organisiertes und verwaltetes Land und Privatbesitz.)

Die durchschnittliche Feldergröße beträgt 1,87 ha. Die Milpas sind in der Regel also sehr klein, zwischen einer und drei Hektaren groß. Der durchschnittliche Familienbesitz beträgt etwa drei Hektaren. Pro Familie (4 Mitglieder) sowie zur Fütterung von einem
Hausschwein und Geflügel genügt die Bebauung einer Hektare zur Selbstversorgung (je eine halbe Hektare bei jedem Zyklus). Der größere Teil der Ernte wird folglich verkauft oder eingetauscht. Dies vor allem gegen Güter wie Öl, Zucker, Kleider, Arbeitsgeräte wie Äxte und Macheten, Kofferradios, Konserven und Alkoholika. Manchmal müssen auch Mais, Bohnen oder Reis hinzugekauft werden.

Nachdem das passende Stück Wald ausgewählt ist, wird mit den Vorbereitungen fürs Pflanzen begonnen. Diese Vorbereitungsarbeiten umfassen das Mähen der Vegetation soweit mit Machete möglich (roza), das Fällen der Bäume (tumba) und das Verbrennen des getrockneten Pflanzenmaterials (quema).

\subsubsection{Roza (Mähen, Roden)}

$\mathrm{Zu}$ Beginn des neuen Jahres (Jan.-Feb.), teilweise auch früher, wenn die Regenfälle etwas abgeklungen sind, beginnt der Bauer damit, auf seinem zukünftigen Feld die Krautvegetation, die Sträucher und dünnstämmigen Bäume (bis zu $25 \mathrm{~cm}$ Durchmesser) mit der Machete umzuhauen. Beim Vorwärtsgehen schafft er sich die gemähte Vegetation mit einem einzackigen Rechen, an dessen Ende ein etwa $30 \mathrm{~cm}$ langer Teil eines Astes stehen gelassen wurde, aus dem Weg.

Gleichzeitig werden die größeren Bäume etwa auf einem Meter Höhe ab Boden rundherum etwa $8 \mathrm{~cm}$ breit geschält und somit zum Absterben gebracht.

\subsubsection{Tumba (Fällen)}

Vom März bis April werden dann die großen Bäume mit Äxten, oder wo vorhanden mit Motorsägen, gefällt. Mittelgroße Bäume werden etwa einen Meter über dem Boden umgeschlagen. Für Urwaldriesen mit breit ausladenden Brettwurzeln baut man ein Brettergerüst, so daß der Baum an einer dünneren Stelle in 2 bis 3 Metern Höhe gefällt werden kann (Abb.2). Zuerst legt man Hand an die größten Bäume und schlägt sie so, daß sie beim Umfallen andere, kleinere Bäume mitreißen. Wo mehr als $15 \mathrm{~m}$ hoher Sekundärwald gerodet wurde, liegen oft noch die Strünke im Maisfeld umher und behindern dessen Bearbeitung. Nachdem die abgestorbene Vegetationsmasse 1 bis 2 Monate zum Trocknen liegen gelassen wurde, kann mit dem Verbrennen begonnen werden.

\subsubsection{Quema (Verbrennen)}

Zum Schutze der umliegenden Waldgebiete und Siedlungen werden Feuerschutzstreifen (guardarrayas) angelegt. Diese sind zwischen 4 und 5 Metern breit. Erst legt man das Feuer gegen den Wind. Ist ein Stück frei, brennt man nun mit dem Wind, damit alles gut verbrannt wird. Im allgemeinen herrscht vorsichtiges Brandroden vor, und klare Grenzen sind ersichtlich. Doch vor allem bei Neusiedlern, die diese Techniken (noch) nicht beherrschen, kommt es oft zu Waldbränden, deren Überwachung und Bekämpfung 
in der Selva Lacandona praktisch aussichtslos ist (Abb. 3).

Mit Abbrennen beginnt man acht Tage nach einem Regen, kurz vor Einsetzen der neuen Regenzeit. 1981 war dies in Sto. Domingo der 10. April (eine Art Bauernkalender scheint bis auf die Mayas zurückzugehen). Infolge der Feuchtigkeit breitet sich das Feuer nur langsam aus, und die kleinen Brände bleiben kontrollierbar. Nicht angekerbte Bäume können das Feuer überdauern. Oft werden nicht alle Bäume gefällt. In einigen Fällen wurden nebst abgestorbenen noch lebende Bäume festgestellt, in einem Fall sogar nur lebende. Die stehengelassenen Bäume halten mit ihren Wurzeln die Erde zusammen, belaubte Bäume geben an zu stark besonnten Stellen noch etwas Schatten. Diese Bäume werden also bewu $\beta t$ stehen gelassen.

Wo Vieh in der Nähe ist, aber auch zum Schutze vor Wildtieren, werden Zäune aufgestellt. Meist verwendet man dazu Holz, das gerodet wurde. Bevorzugt werden Hölzer mit Dornen (z. B. Akazien wie "cuernos de torro", Acacia sp.). Oft schlagen die Pfähle wieder aus, und es entstehen Hecken. Wo die Gefahr von einbrechenden Rinderherden besteht, wird meist Stacheldraht verwendet.

\subsubsection{Siembra (Stecken)}

Mit dem Stecken von Mais kann schon ein bis zwei Tage nach dem Brandroden begonnen werden. Bis spätestens zum 15. Mai müssen die Körner im Boden sein, da dann die Regenzeit einsetzt und die Körner ausgeschwemmt würden. Gesteckt wird, «wenn der Mond günstig ist». 1981 war dies bei zunehmendem Mond am 10. Mai der Fall. Alle befragten Bauern hatten den Mais an diesem Tag gepflanzt. Bei Nichtbefolgen dieser Regel würde die Maisernte gefährdet, wird von den Bauern erklärt. Mit einem einfachen Holzstecken (palo) wird ein etwa $10 \mathrm{~cm}$ tiefes Loch in den Boden gestoßen, und zwischen 5 und 8 Körner werden hineingegeben (die Mehrzahl der Bauern steckt 7 Körner). Es werden mehrere Körner ins selbe Loch gegeben, da nicht alle keimen werden. So erhält man vier und mehr Maisstengel, die sich gegenseitig etwas stützen und so weniger leicht vom Wind geknickt werden. Die Samen werden nicht zugedeckt, und der Boden wird somit wenig umgearbeitet. Gepflanzt wird, wo dies möglich ist, in Reihen. Diese, wie auch die Pflanzen innerhalb der Reihe, liegen zwischen $80 \mathrm{~cm}$ und $2 \mathrm{~m}$ auseinander. Abstände von $1,20 \mathrm{~m}$ bis $1,60 \mathrm{~m}$ sind die Regel. Pro Hektare werden 15 bis $25 \mathrm{~kg}$ Maiskörner, je nach Abständen und Anzahl Körner pro Loch, gesteckt.

Die verbreitetste Maissorte ist maiz amarillo (gelbkörniger Mais), der als etwas hart zu mahlen gilt. Die Sortenvielfalt, wie sie noch im Hochland von Chiapas anzutreffen ist, ging verloren. Diese Einheitshybride ist viel anfälliger für Krankheiten und Schädlinge, als dies eine Vielzahl von verschiedenen Sorten wie maiz

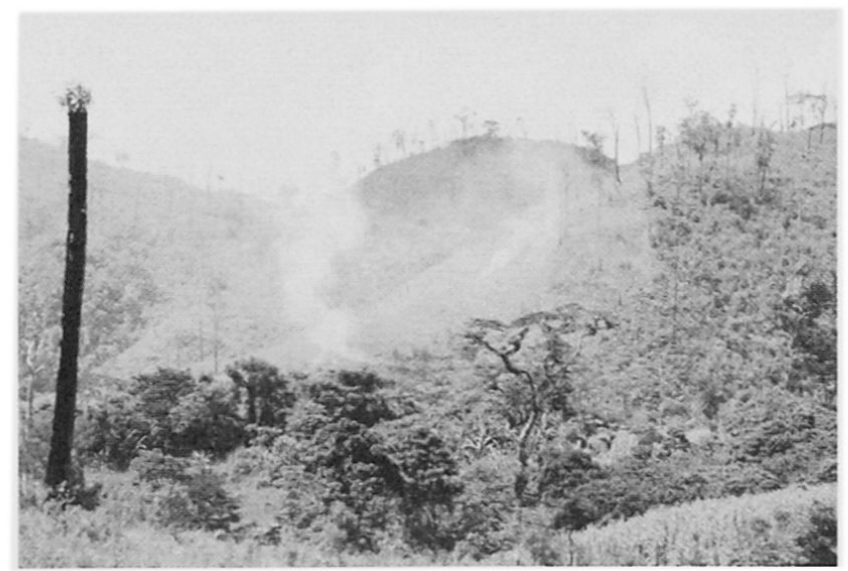

Abb. 3 In einem bereits vollständig abgeholzten Gebiet bei Chancalá wird das getrocknete Pflanzenmaterial einer Parzelle verbrannt. Im Hintergrund sind weitere vorbereitete Milpas, vorne rechts ist eine Milpa mit reifem Mais zu erkennen (Nov. 81).

cuarentaño, diente de perro, maiz negro, maiz blanco oder maiz colorado wäre. Gleichzeitig (oder ein paar Tage früher, damit die Keimblätter beim Stecken des Maises schon da sind) werden die Bohnen in die Reihen zwischen die Maispflanzen gesteckt. Pro Loch verwendet man etwa vier Bohnen. Andere Bauern stecken vier Maiskörner und ein bis zwei Bohnen zusammen ins selbe Loch. Die Bohnen werden an den Maisstengeln emporranken. Wo noch Baumstämme liegen, werden die Bohnen in 15 bis $20 \mathrm{~cm}$ Abstand davor gepflanzt und klettern dann an diesen empor. Wo eine Monokultur mit Bohnen angelegt wird, stehen die Stauden $40 \times 60 \mathrm{~cm}$ weit auseinander. Pro Hektare muß 15-20 kg Saatgut an Bohnen verwendet werden. Sollen zwischen dem Mais Kalebassen gezogen werden, genügt $1 \mathrm{~kg}$ pro Hektare (2-3 m Abstand zwischen den einzelnen Pflanzen). Bohnen und Kalebassen steckt meist ein Knabe.

\section{Die Anbauprodukte}

Hauptanbauprodukte sind Mais (Zea mays L.) und Bohnen (Phaseolus sp.). Diese sind zugleich Hauptnahrungsmittel. Die Maiskörner werden gemahlen und in etwas Wasser zu einem Brei angerührt (posol), aber hauptsächlich zu Maisfladen (tortillas) verarbeitet. Die Bohnen werden gekocht und danach meist zerstoßen als frijol gegessen. Neben Mais und Bohnen werden noch eine Vielzahl weiterer Kulturpflanzen angebaut. So können z. B. Mais und Kürbis (Cucurbita pepo L. und maxima Duch.), Mais und Sesam (Sesamum indicum) oder Bohnen und Reis (Oryza sativa L.) zusammen angebaut werden. Zudem werden in geringerem Maße Bananen (Musa sp.) Yuca = Manjok (Manihot esculenta), Camote (Süsskartoffel, Ipomoea batatas), Macal (Knollen einer riesenblättrigen Araceae), Zuckerrohr (Saccharum officinarum L.), Chile (Capsicum frutescens L.), Tomaten (Lycopersicon lycopersicum (L. Farw.) und Zwiebeln (Allium cepa L.) gepflanzt. Die wenigen Lacandonen-Indianer imitie- 
ren die Vielfalt des Waldes und pflanzen viele verschiedene Produkte zerstreut an. Dies hat zur Folge, daß weniger Krankheiten und weniger Erosion auftreten. Einige Pflanzen werden nur angebaut, um Tiere anzulocken und in der Milpa zu jagen.

Eine Vielzahl von Krankheiten und Schädlingen bedrohen vor allem die Monokulturen. Beim Mais sind dies Vögel, Dachse und Waschbären, welche die Körner fressen, und Tepezcuintles (kleine, hirschartige Tiere), die die ganzen Pflanzen abfressen. Ratten sind besonders bei gelagertem Mais eine Bedrohung. Die größeren Tiere werden gejagt (z. T. werden Fallen gestellt). Pestizide werden aus Kosten- und Transportgründen keine verwendet. Kunstdünger werden im allgemeinen nicht verwendet.

\subsubsection{Limpia (Jäten/Säub乞rn)}

Zweimal während der Vegetationszeit des Maises wird "gejätet». Dies kann jedoch nie geschehen, wenn es trocken ist, denn dadurch würde der Boden Feuchtigkeit verlieren, und die Temperaturwechsel des Bodens würden sich negativ auf die Pflanzen auswirken. Zudem könnten sich Konkretionen von Eisen und Magnesium bilden.

Die erste Säuberung von Unkraut erfolgt etwa 15-20 Tage nach der Keimung. Mit der Machete, ausnahmsweise mit der Sichel (azadón), werden die nicht gewünschten Kräuter abgemäht. Dieser Vorgang heißt auch chapoleo. Da die Pflanzen nicht ausgezerrt werden, verbleiben die Wurzeln im Boden und schützen diesen vor Erosion. Das Abgemähte wird liegen gelassen und schützt damit den Boden vor dem Austrocknen und den heftigen Regengüssen. Zudem wird der Boden so auf natürliche Weise etwas gedüngt. Das zweite «Jäten» erfolgt vor der dobla, dem Knicken der Maisstengel.

Oftmals wird dem «Jäten» eine geringere Bedeutung zugemessen oder sogar ganz darauf verzichtet. Zur Zeit der dobla verschwinden die Maispflanzen in einem Gewirr von aufkommenden Gramineen etc., so daß der Eindruck einer Wiese entsteht (Abb.4).

Bei Polykulturen mit Kalebassen ist weniger Säubern notwendig, da diese Pflanzen mit ihren großen Blättern den Boden gut überdecken.

\subsubsection{Dobla (Knicken)}

Damit die Maiskörner voll ausreifen können und die Kolben nicht alle zusammen im selben Zeitpunkt geerntet werden müssen, werden die Stengel der Maispflanzen unterhalb des Kolbens geknickt (Abb.4). Dadurch hängen die Kolben nach unten; es dringt keine Feuchtigkeit ein, und Pilzbefall wird so verhindert. Zudem sind die Körner so besser vor den Vögeln geschützt. Die dobla erfolgt etwa zwischen dem 15. und 20. August, drei Monate nach dem Stecken. Geknickt wird mit dem stumpfen Teil der Machete, 1/4 unterhalb des Kolbens. Kümmerliche Pflanzen werden nicht geknickt.

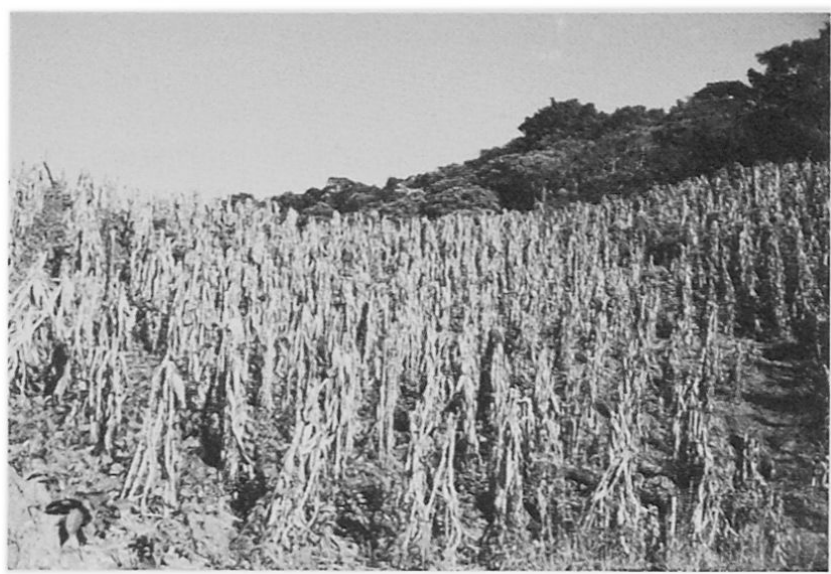

Abb. 4 Geknickter Mais. Die Milpa befindet sich auf ca. 800 m ü. M., zwischen Palenque und Ocosingo (Nov. 81).

\subsubsection{Cosecha (Ernte)}

Etwa ein Monat nach dem Knicken, zwischen September und Oktober, wird mit der Ernte begonnen. Kurz zuvor wurde ein einfacher Unterstand (troje) gebaut, unter welchem die Kolben zunächst zum Trocknen aufgehäuft werden. Später werden sie zum Wohnhaus transportiert und dort unter einem Dach gelagert. Die Kolben werden von Hand und mit den Lieschblättern abgebrochen. Die Lieschblặtter werden erst später entfernt. Zur Erntezeit können Hilfskräfte eingestellt werden, welche 50 Pesos (1979) pro Tag verdienen. Die abgeernteten Maisstengel werden stehen gelassen. Der Mais wird meist je nach Bedarf geerntet, und somit können die Kolben bis in den März hinein an den Pflanzen verbleiben.

Die Ernte der Bohnen erfolgt, wenn die Pflanzen trocken sind. Will man diesen Zustand früher erreichen, werden die Blätter abgezupft. Die Ernte findet meist im September statt. Die getrockneten Bohnen werden in Zuckersäcken aufbewahrt.

\subsubsection{Desgrano (Entkörnen)}

Die Kolben werden unter einem Vordach entkörnt. Häufig macht dies ein alter Mann. Zerfressene Körner usw. werden als Viehfutter ausgeschieden, die leeren Kolben zum Feuern verwendet. Ist der Mais zum Verkauf bestimmt, werden die Körner in $50-\mathrm{kg}$ Säcke abgefüllt (400 Kolben ergeben ca. 60 kg Mais). Durch unsachgemäße Lagerhaltung geht leider ein ansehnlicher Teil der Ernte verloren (2).

\subsection{Der Anbauzyklus «milpa de tornamil» (Abb. 5)}

Das Fällen und das Brennen entfallen in diesem Zyklus. Nachdem das Feld der "milpa de año" abgeerntet ist, wird der Boden für den neuen Zyklus vorbereitet und mit der Machete gesäubert. Die bestellte Fläche während dieses Zyklus ist meist kleiner als während der «milpa de año». Oftmals 
werden die Maisstengel der «milpa de año» stehen gelassen und dienen den neu gepflanzten Bohnen als Kletterstütze. Die Bohnen werden Ende November gesteckt, der neue Mais anfangs Dezember. So werden die Nordwinde (nortes) ausgenützt. Gejätet wird wiederum ein- bis zweimal, das erste Mal, nachdem der Mais etwa $40 \mathrm{~cm}$ hoch ist, das zweite Mal vor der "dobla». Geknickt wird der Mais im April. Die Ernte erfolgt sechs Monate nach dem Stecken, also von Mai bis Juli. Die Bohnen können schon im Februar/März geerntet werden. Die ausgeführten Arbeiten unterscheiden sich nicht von denen der "milpa de año».

\subsection{Der Aufbauzyklus des «canigular» (Abb. 5)}

Weit seltener wird noch in einem weiteren Zyklus angebaut, dem "canigular» (den sog. Hundstagen). Auf einer kleinen Fläche wird drei bis zehn Monate alte Sekundärvegetation (acahual) mit der Machete gemäht und werden Bohnen gesteckt (Juli/August). Geerntet wird im November/Dezember.

\subsection{Produktivität}

\subsubsection{Arbeitsaufwand}

Für die Bewirtschaftung einer Milpa im Zyklus «milpa de año" müssen ca. 70-80 Manntage pro Hektare an Arbeit aufgewendet werden. Da im Zyklus "tornamil»" die "tumba" und die "quema" entfallen und die anderen Arbeiten auch weniger Zeit beanspruchen, werden hier etwa 30 Manntage benötigt. Total müssen also pro Hektare und Jahr etwa 110 Manntage an Arbeit investiert werden. Drei Hektaren stellen also für einen einzelnen Mann das Maximum dar, das er allein bewirtschaften kann (für genauere Angaben siehe TURRENT-FERNANDEZ 1978 oder GELBERT 1982).

\subsubsection{Hektarertrag}

Die durchschnittlichen Hektarerträge für Mexiko betragen bei Mais 1961/65 10,6 t/ha und 1972 11,5 t/ha (FRANKE 1976). Die Erträge der beiden Anbauzyklen "milpa de año" und "tornamil" fallen recht unterschiedlich aus. So können die der "milpa de año» bis doppelt so groß sein wie diejenigen des "tornamil». Beispielsweise beträgt der Hektarertrag in der "Sierra de Madrigal», Tabasco, bei der "milpa de año» 1,5-2 t, beim "tornamil" zwischen 500 und $1000 \mathrm{~kg}$. In der Selva Lacandona schwanken die Erträge zwischen $0,97 \mathrm{t} / \mathrm{ha}$ (Nuevo Huistán) und 12,5t/ha (Boca del Cerro). Den Lacandonen werden Erträge zwischen 2,8 t/ha (Fallstudie Mpio. Las Margaritas 1975) und $6 \mathrm{t} /$ ha (NATIONS 1981) zugeschrieben. Bei den Bohnen schwanken die durchschnittlichen Erträge für Mexiko zwischen 4,2 t/ha (1961/65 und 5,2 t/ha (1972). Der Weltdurchschnitt beträgt 19725 t/ha (FRANKE 1976). In

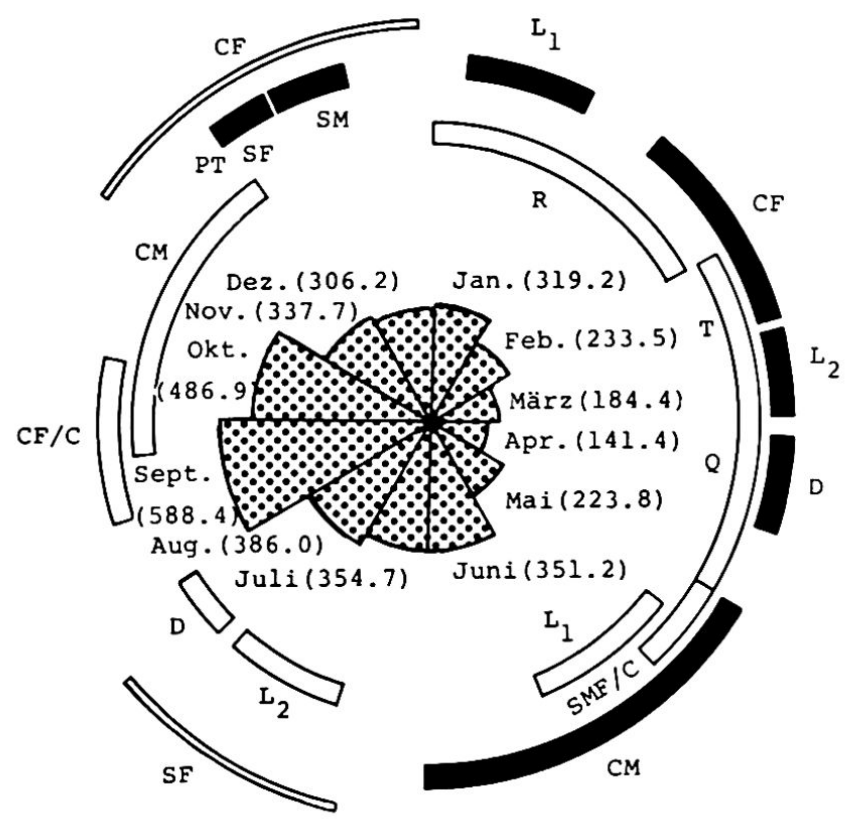

Abb. 5 Anfallende Arbeiten in den drei Anbauzyklen und Niederschlagsverteilung.

Milpa de Año (Anbau gleichzeitig und am selben Ort, Mais, Bohnen, Kalebassen

- Tornamil (Anbau zu verschiedenen Zeiten, am selben Ort, Bohnen und Kalebassen

$\square$ Zyklus des «Canigular»

• Niederschläge in $\mathrm{mm}$

R: Roza (Roden/Mähen)

T: $\quad$ Tumba (Fällen der Bäume)

Q: Quema (Abbrennen des getrockneten Pflanzenmaterials)

SMF/C: Siembra de Maiz, Frijol y Calabaza (Stecken der Maiskörner, Bohnen und Kalebassensamen)

$L_{1}, L_{2}:$ Limpia (Jäten)

D: $\quad$ Dobla (Knicken)

CM: Cosecha de Maiz (Maisernte)

CF/C: Cosecha de Frijol y Calabaza (Ernte der Bohnen und Kalebassen)

PT: $\quad$ Preparación del Terreno (Vorbereiten des Feldes)

SF: $\quad$ Siembra Frijol (Stecken der Bohnen)

SM: $\quad$ Siembra Maiz (Stecken der Maiskörner)

CF: Cosecha Frijol (Ernte der Bohnen)

der Selva Lacandona fallen die Erträge geringer aus, doch gilt es hier zu berücksichtigen, daß es sich nicht um mit viel Technologie bewirtschaftete Monokulturen handelt. 400-600 kg/ha Bohnen können als durchschnittliche Erträge angesehen werden (BRAUER 1981).

\subsubsection{Ertragsrückgang oder Ertragszunahme im zweiten Anbaujahr}

Im allgemeinen sinken die Erträge nach einiger Zeit rapide ab. Das erste Anbaujahr gilt als das ertragsreichste. Danach fallen die Erträge immer geringer aus. In Guatemala z. B. sinkt die Maisproduktion von $100 \%$ im ersten Jahr auf $50 \%$ im zweiten Jahr (WEI- 
SCHET 1979). Die bei der Brandrodung frei gewordenen Mineralstoffe, die als Asche dem Boden aufliegen, düngen diesen zwar anfänglich und verbessern die Kultivierung. Doch mit den heftigen Regengüssen werden sie sehr schnell abgeschwemmt und gehen dem Boden verloren. Aus diesem Grund muß das Feld verlassen und eine neue Parzelle gerodet werden. Dieser allgemein bekannten Tatsache scheint jedoch eine Beobachtung zu widersprechen, die mir mein Hauptinformant Jerónimo, in Sto. Domingo schilderte. Danach fallen bei gerodetem Primärwald die Erträge im ersten Jahr befriedigend aus. Im zweiten Jahr jedoch steigen sie an, um erst im darauffolgenden Jahr beträchtlich abzunehmen, worauf das Feld verlassen wird. Die Erklärung für dieses Phänomen scheint zu sein, dass die Mineralstoffe der Asche und diejenigen der Tiefenerosion kurz nach dem Brandroden wegen im Boden verbliebener Wurzeln die Kulturpflanzen nicht vollständig erreichen, sondern erst im darauffolgenden zweiten Jahr (Abb. 6). Zudem behindern die Wurzeln großer Bäume die Ausbreitung der Wurzeln der Kulturpflanzen. Beim Abbrennen von Primärwald wird nur ein Teil der Mineralstoffe zu Asche. Ein großer Teil wird erst nach dem Zerfallen der nicht verbrannten Baumstrünke frei. Bei
Sekundärwald verbrennt wohl ein größerer Teil der Vegetationsmasse und wird zu Asche, ein größerer Teil der Mineralstoffe erreicht die Wurzeln der Kulturpflanzen schon im ersten Anbaujahr. Schließlich werden sich auch die Unterschiede bei der floristischen Zusammensetzung zwischen Primär- und (auch älterem) Sekundärwald auswirken (SARUKHAN 1964).

\subsubsection{Dauer der Bewirtschaftung}

Die Dauer der Bewirtschaftung einer Parzelle bewegt sich in der Selva Lacandona zwischen einem Jahr und vier Jahren. Durchschnittlich und in den meisten Fällen erfolgt das Shifting nach zwei Jahren. In den Ebenen und in den bewaldeten Gebieten des Flachlandes können die Felder im allgemeinen länger bewirtschaftet werden als an anderen Stellen.

\subsubsection{Feldverlegungsmuster}

Feldverlegungsmuster, wie sie z. B. SPENCER (1966) aus SE-Asien beschreibt, ließen sich im Untersuchungsgebiet nicht erkennen. Bei der Befragung konnten sich die Bauern auch jeweils höchstens an das letzte Feld erinnern (siehe auch Kap. 1.1.1). Wo

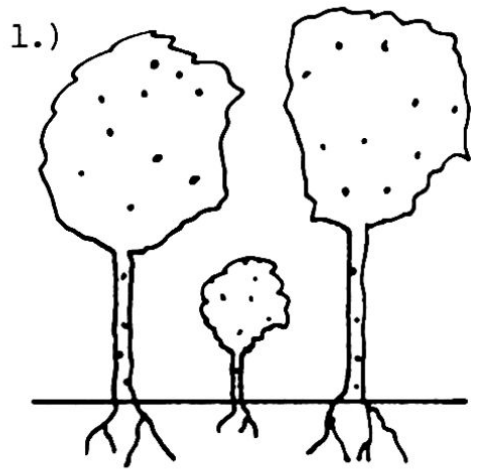

Mineralstoffe, in Vegetation gespeichert

\section{2.)}

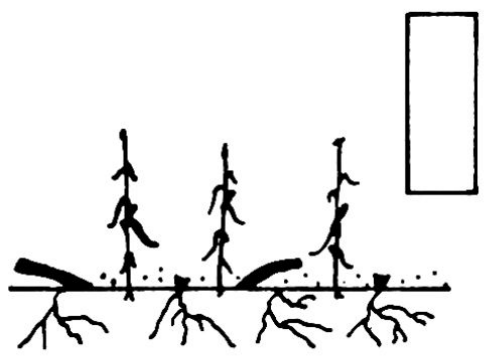

1. Anbaujahr: Mineralstoffe liegen dem Boden als Asche auf. Verbliebene Wurzeln erlauben nur zögernd deren Eindringen in den Boden und behindern Wurzeln des Mais.

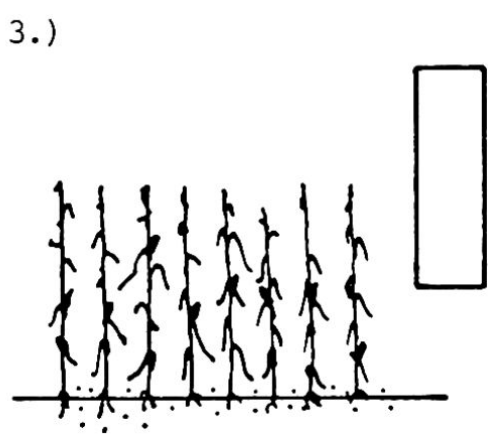

2. Anbaujahr: Mineralstoffe sind z. T. in den Boden eingedrungen und wirken als Dünger. Zerfallende Baumstrünke geben Mineralstoffe frei.

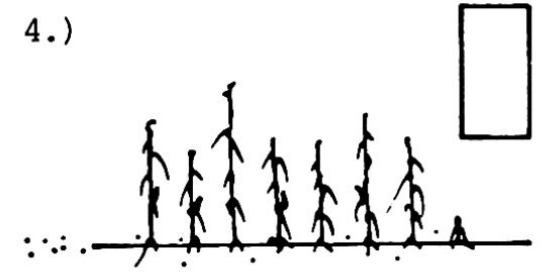

3. Anbaujahr: Dem Boden kann kein neuer "Dünger" zugeführt werden, da der Regen die Asche weggespült hat.
5.)

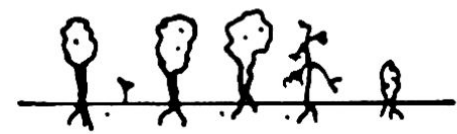

Nach dem 3. Anbaujahr: Das Feld wird aufgegeben, Sekundärvegetation kommt hoch.

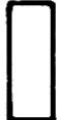

relativer Ertrag Mineralstoffe

Entgegen der weitverbreiteten Ansicht einer kontinuierlichen Ertragsabnahme vom ersten Jahr an fällt in Sto. Domingo der Ertrag auf Flächen gerodeten Primärwaldes im zweiten Anbaujahr grösser aus als im ersten.

Abb. 6 Ertragszunahme im zweiten Anbaujahr. 
dies aus Gründen der Morphologie, der Vegetation und der Besitzverhältnisse möglich ist, trachtet der Bauer danach, sein Feld a) möglichst nahe bei seinem Wohnhaus, b) möglichst in der Nähe der Verkehrswege und c) an die alte Milpa anschließend anzulegen. Ist letzteres der Fall, lassen sich bestehende Trampelpfade und Wege ausnützen, es dringen von einer Seite her weniger Unkräuter ein, und die Parzelle erhält unter Umständen mehr Licht. Liegt das Wohnhaus in der Nähe der alten Milpa, muß es beim «Shifting» nicht auch noch verlegt und damit neu gebaut werden.

Auf ein interessantes Feldverlegungsmuster stie $B$ ich in Sto. Domingo. Der Bauer und «Restaurant»-Besitzer Jerónimo praktiziert hier eine Vier-Felder-Wirtschaft mit einjähriger Bebauung derselben Milpa. Das «Shifting» findet also innerhalb eines bestimmten Territoriums statt, das wohl wegen des Bevölkerungsdruckes nicht verlegt werden kann.

Im ersten Jahr wird die 1 ha große Milpa in einer Ecke der 4,5 ha großen Besitzung angelegt. Im nächsten Jahr wechselt der Bauer zur gegenüberliegenden Seite. Im dritten Jahr zieht er in die anschließende Ecke, im folgenden Jahr in deren gegenüberliegende Ecke usw. Jede verlassene Milpa liegt somit mindestens drei Jahre brach, kann dafür aber nur ein Jahr lang bewirtschaftet werden.

\subsubsection{Hausbau/Siedlungen}

Die Behausungen derjenigen Bauern, die noch eine «echte» Shifting Cultivation praktizieren, d.h. ihr Haus bei Feldverlegungen periodisch ebenfalls neu erstellen, sind recht einfach. Die einräumigen Behausungen bestehen meist aus einem Balkengerüst, Knüppel- oder Rohrwänden und einem Palmblattdach. Die Materialien zum Bau liefert die Umgebung. Palmen sind Teil der Regenwaldvegetation (z. B. Coroz-Palmen, Orbignya cohune), und Spanisch-Rohr wird z. T. gepflanzt. Lianen ersetzen die hier sowieso ungeeigneten Nägel. Dieser Haustyp hat sich aus dem Mayahaus entwickelt und ist heute noch in seiner Grundstruktur weit nach Yucatan und nach Guatemala hinein verbreitet. Bei Bevölkerungsdruck und damit verbunden bei permanentem Wohnen werden die Häuser stabiler gebaut. Meist errichtete man eine kleine Fundamentmauer aus Beton und zementiert den Fußboden. Die Bretterwände bestehen vielfach aus Edelholz (z. B. Mahagony, Chicozapote etc.). Das Dach wird mit Wellblech oder Dachpappe gedeckt. Da es im Interesse der Bauern liegt, sich zusammenzuschließen, um in den Genuß staatlicher Investitionen zu gelangen, entstehen immer mehr eigentliche Dörfer (Straßen- und Haufendörfer).

\subsubsection{Aufgabe des Feldes}

Nachdem das Feld zum letzten Mal abgeerntet wurde, bleibt es der Natur überlassen. Allenfalls wird die Milpa noch durch eine Bananenpflanzung für weitere
18 Monate genutzt, die eingedrungenen Kräuter und kleinen Bäume werden aber nicht mehr gemäht. Der zukünftige acahual (Sekundärwald) wird vom Bauern im allgemeinen nicht aktiv beeinflußt, d. h. es werden keine Samen ausgestreut oder Baumsetzlinge gepflanzt. Eine Ausnahme sei hier jedoch noch erwähnt: In einigen Gebieten säen die Bauern nach dem Verlassen des Feldes eine Leguminose aus, die Café Nez oder Nescafé genannt wird. Diese Pflanze breitet sich sehr schnell aus und überdeckt alle anderen Pflanzen, die dadurch ersticken. Café Nez bringt Stickstoffe in den Boden und übt so eine düngende Wirkung aus. Die Hülsen werden abgelesen, und aus den Bohnen wird ein kaffeeähnliches Getränk gebraut. Die Nescafé-Bohnen gelangen nicht in den Handel. Soll nach einiger Zeit der Brache das Feld neu bestellt werden, können die Pflanzen leicht mit einem Machetenhieb knapp über dem Boden durchtrennt und zum Absterben gebracht werden.

Die Regeneration des Waldes wird von den in der Erde verbliebenen Wurzelstöcken und von den in der Milpa geduldeten Unkräutern mitbeeinflußt. SARUK. HAN (1964) stellt fest, daß die floristische Zusammensetzung verschieden ist, je nach Zeitpunkt der Rodung und Alter des Sekundärwaldes.

\subsection{Huertos familiares (Familiengärten/Hausgärten)}

Im allgemeinen besitzt jede Familie einen Hausgarten in der Größe von bis zu einer halben Hektare. Dieser Garten wird zum Schutz gegen wilde Tiere, oder wenn Gemüse angebaut wird, gegen Schweine, eingezäunt. Der huerto familiar befindet sich möglichst in der Nähe des Hauses, meist steht das Haus sogar mitten im Garten. Der Hausgarten versorgt die Familie mit Gemüse, Früchten, Gewürzen und Heilkräutern. Daneben werden auch Zierpflanzen gezogen. Beim Roden für den Platz des Wohnhauses werden natürlich vorkommende Fruchtbäume geschont und stehen gelassen.

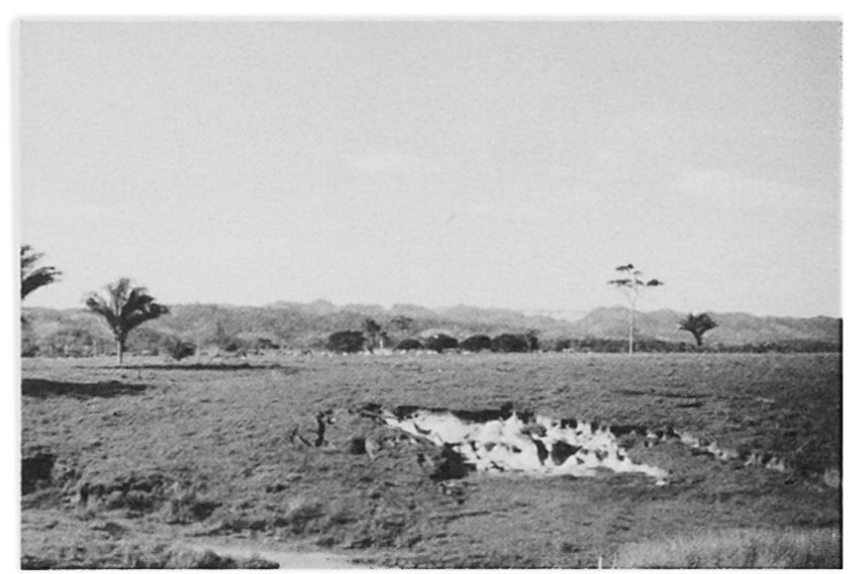

Abb. 7 Als Folge des Rodens des tropischen Regenwaldes und der Viehwirtschaft treten bereits schwere Erosionsschäden auf (Lacandón, 9.9.81). 


\subsection{Weitere wirtschaftliche Tätigkeiten}

Neben dem Anbau von Nahrungsmitteln spielt auch das Sammeln, zumindest in einigen Gebieten, noch eine gewisse Rolle. Drei Pflanzen sind dabei von Bedeutung: Chicle (Kaugummimasse), Shate (Blätter von Chamaedorea $s p$. für Dekorationszwecke und Kränze vor allem in den USA) und Barbasco (Dioscorea composita, zur Gewinnung von Östrogen). Chicle und Barbasco sind heute durch die synthetische Herstellung von Ersatzprodukten bedroht.

\section{Waldveränderungen in der Selva Lacandona}

Das noch vor wenigen Jahrzehnten homogen dicht bewaldete Gebiet der Selva Lacandona bietet heute ein Bild maßloser Zerstörung. Weite Landstriche würde man heute treffender als "Pastizál Lacandon» (Weideland) denn als "Selva» Lacandona bezeichnen. Die Zerstörungen schreiten rasch vorwärts. Mit jeder neu gebauten Straße erhält die Kolonisation Einlaß in ein bisher noch einigermaßen verschont gebliebenes Gebiet.

Der größte Teil der Bevölkerung der Selva Lacandona betreibt in der Form von Shifting Cultivation Landwirtschaft zur Selbstversorgung. Durch den großen Bevölkerungsdruck (die Bevölkerung der Selva Lacandona hat sich von 1974 bis 1980 v. a. wegen Einwanderungen aus dem Hochland von Chiapas verdoppelt und betrug 1981 nach Schätzungen der Secretaria Forestál, Palenque, mehr als 100000 Personen) wird bebaubares Land immer seltener, und als Folge davon verkürzen sich die Brachezeiten. Die Fläche der Selva Lacandona beträgt 1308312 Hektaren. Davon waren ursprünglich $84 \%$ bewaldet. 1970 wurden 60000 ha landwirtschaftlich genutzt (inkl. Viehzucht). Fünf Jahre später waren es bereits 153000 ha. Darin nicht eingeschlossen sind 140000 ha quemadales (aufgegebene Brandrodungen, Sekundärwälder ohne Caobas). Bis 1980 nahm die Fläche landwirtschaftlicher Nutzung nochmals um 100000 ha auf 253000 ha zu. Rechnet man die schätzungsweise brachliegenden 200000 ha dazu, ist die Zahl der 470000 ha, die von der Forstbehörde als überhaupt landwirtschaftlich nutzbar angesehen werden, bereits überschritten (Quelle: Secretaria Forestál, Palenque). Immer mehr zeichnet sich eine Tendenz zur Viehzucht ab. Bereits ein Viertel der in der Landwirtschaft tätigen Bevölkerung betreibt Viehzucht (genauere Angaben sind leider nicht erhältlich). Sobald sich die neuen Siedler nach ein paar Jahren etabliert haben, beginnen sie vom Maisbau auf Viehzucht umzustellen, und die Rodungen werden $\mathrm{zu}$ endgültigen Wunden in der Landwirtschaft (Abb.3). Als Folge davon ist schon vielerorts verstärkte Erosion zu beobachten (Abb. 7). Früher klares Wasser führende Flüsse sind heute braun.

\subsection{Das Verhältnis der Bevölkerung zum Wald}

Die heutige Bevölkerung der Selva Lacandona hat meiner Meinung nach ein gestörtes Verhältnis zum Wald. Dies wirkt sich wegen des großen Bevölkerungsdruckes verheerend auf die Umwelt aus. Sogar die Behörden sind sich des «totalen Desinteresses der Bevölkerung an der Erhaltung der forstwirtschaftlichen Ressourcen» bewußt (ACOPA/CALLEROS 1981). Die meisten Kolonisten haben sich erst seit kurzem in der Selva Lacandona niedergelassen und bringen Kenntnisse einer anderen Umwelt mit. Für sie stellt die Selva in jeder Beziehung Neuland dar. Wald ist für sie hier scheinbar unerschöpflich vorhanden. Ein großer Teil der Siedler stammt aus dem Hochland von Chiapas, wo zwar schon schlimme Erosionserscheinungen sichtbar sind, aber auch aufgeforstet wird.

Besonders für Viehzüchter stellt Wald ein Übel dar, das verschwinden muß. "Für den Viehzüchter hat der Wald einen geringen oder gar keinen Wert, da für ihn die Alternative heißt: Wald oder Weide. Die Entscheidung fällt ihm deshalb nicht schwer» (Proyecto Selva Lacandona, 1975/76). Weideland ist im Gegensatz zu Wald «sauber» (limpio).

Ein Dekret vom 12. Januar 1978 schützt die Selva Lacandona ausdrücklich. Und eine riesige, von der UNESCO geförderte Schutzzone, die «Reserva Integral de la Biosfera», besteht schon lange auf dem Papier, wird aber nicht in die Tat umgesetzt. Wird es dazu einmal zu spät sein?

\section{Mögliche Maßnahmen zum Schutz der Selva Lacandona}

Die gegenwärtige Entwicklung zunehmender Zerstörungen, die in nächster Zukunft zum gänzlichen Auslöschen des tropischen Regenwaldes in der Selva Lacandona führen werden, muß gebremst oder ganz gestoppt werden, damit die ansäßige Bevölkerung weiterhin und langfristig ihren Nutzen aus dem Gebiet und seinen Ressourcen ziehen kann. Folgende Maßnahmen empfehlen sich:

\subsection{Totaler Einwanderungsstop für die Selva Lacandona}

Voraussetzung für einen wirkungsvollen Zuwanderungsstop ist die Kenntnis genauer Bevölkerungszahlen. Diese sollten innerhalb möglichst kurzer Intervalle neu erhoben werden. Gleichzeitig müssen in anderen Landesteilen geeignete Maßnahmen, so z. B. eine Landreform im Hochland von Chiapas, getroffen werden, damit es nicht zu sozialen und ethnischen Unruhen kommt (über die Durchführbarkeit dieser Maßnahmen mache ich mir keine Illusionen). 


\subsection{Landesnutzungsplanung}

Aufgrund des Forstinventars und weiterer wissenschaftlicher Untersuchungen soll eine Landnutzungsplanung vorgenommen werden. Eine solche Planung darf aber nicht bloß aus einer Anlehnung an die aktuelle Landnutzung bestehen. So darf Viehzucht an völlig ungeeigneten Stellen nicht einfach deshalb toleriert werden, weil sie sich dort bereits etabliert hat.

\subsection{Gesetzgeberische Maßnahmen}

\subsubsection{Beschränkung der Viehzucht}

- Höhere Stückzahlen dürfen nicht zugelassen werden. Dies gilt besondes für Hornvieh.

- Aus (Erosions-)gefährdeten Gebieten muß die Viehzucht gänzlich verbannt werden.

- Ein striktes Weideverbot an Hängen mit mehr als $15 \%$ Steigung muß durchgesetzt werden.

\subsubsection{Schaffung von absoluten Schutzgebieten für Flora und Fauna}

Diese sollten sich nicht nur auf Rückzugsgebiete beschränken. Solche Schutzgebiete sollten wirkungsvoll geschützt werden. In der (auf dem Papier) bestehenden Schutzzone "Reserva Integral de la Biosfera» ist bis heute der Schutz des Waldes nicht gewährleistet.

\subsubsection{Schutz gewisser Baumarten}

Es muß verboten werden, gewisse gefährdete Arten (wie z. B. die Caoba, Swietenia macrophylla) zu fällen.

\subsubsection{Durchsetzung des Jagdverbotes}

Gewisse gefährdete Tierarten müssen geschützt und Vergehen schwer bestraft werden (leider werden einem z.B. immer wieder Jaguarfelle zum Kauf angeboten, obwohl ein Jagdverbot dieser Tierart besteht!).

Die bestehenden Gesetze müssen endlich durchgesetzt und überwacht, Verstöße geahndet werden.

\subsection{Forstwirtschaftliche Maßnahmen}

\subsubsection{Forstinventar}

Um gewisse Gebiete zu Schutzzonen erklären zu können und um die zukünftige Landnutzung zu planen, muß ein aktuelles Forstinventar erstellt und in möglichst kurzen Intervallen nachgetragen werden. Die Ausführung eines solchen Projektes könnte der DETENAL (Dirección de Estudios del Territorio Nacionál) übertragen werden, welche mit Fernerkundungsmethoden arbeitet. Die lokale Forstbehörde verfügt über besonders nützliche Kenntnisse und
Verbindungen und wird deshalb ebenfalls am Projekt eines solchen Forstinventars zu beteiligen sein.

\subsubsection{Schaffung von lokalen Forstkreisen}

Die Verantwortung für den Wald sollte den einzelnen Gemeinden oder Ejidos übertragen werden. Dazu müssen Förster und Förstergehilfen ausgebildet werden, die von den Gemeinden und Ejidos gestellt werden.

\subsubsection{Wiederaufforstungen}

Leider scheint es beispielsweise bis heute unmöglich zu sein, Mahagony wiederaufzuforsten, da die Keimfähigkeit der Samen anscheinend bereits nach 2-3 Tagen erlischt. Der Artenreichtum des tropischen Regenwaldes und die verschiedenen Anbaubedingungen für jede Baumart erschweren Wiederaufforstungen. Frau DUBY-BLOM sagte (1981): «The trouble is that we can reforest only in the highlands of Chiapas. The pine will grow almost anyplace.» Sicherlich lassen sich einmal in naher Zukunft Erfolge mit Wiederaufforstungen einheimischer Bäume erzielen, wenn Anstrengungen in dieser Richtung unternommen werden.

\subsubsection{Breitere, nicht selektive Nutzung des Waldes}

Neben den Hartholzbäumen lassen sich auch andere Bäume auf irgend eine Weise nutzen (siehe z.B. GELBERT 1982, p. 106). Kleinindustrielle Betriebe sollten an Ort und Stelle das Holz zu Möbeln, Werkzeuggriffen usw. weiterverarbeiten.

\subsection{Verbesserung der Landnutzung}

\subsubsection{Intensivierung der Viehwirtschaft}

Eine Intensivierung der Viehzucht zwecks Abnahme der Flächenbedürfnisse (von gegenwärtig 2,7 ha) pro Tier wäre sicher zu einem großen Teil mit geordneten Weidemaßnahmen zu erreichen.

\subsubsection{Alternative Landnutzungsmethoden}

Die Lacandonen pflegten jeweils verschiedene Pflanzen, sowohl Kulturpflanzen als auch Pflanzen, um jagdbare Tiere anzulocken, in der selben Milpa anzubauen. Dadurch wurde der Boden nicht einseitig ausgelaugt, Erosionsschäden hielten sich in Grenzen, und es ging weniger durch Tierfraß verloren.

Die Neusiedler kennen nur eine beschränkte Anzahl von Nutzpflanzen aus dem tropischen Regenwald. $\mathrm{Zu}$ $80 \%$ (!) bauen sie Monokulturen an, deren Erträge geringer als bei den Lacandonen ausfallen. Das über Jahrhunderte angeeignete Wissen der Lacandonen sollte nicht einfach verlorengehen, sondern sinnvoll $\mathrm{zu}$ beiderseitigem Nutzen verwendet werden. In diesem Sinne könnte der Bildungsstand der einheimi- 
schen Bevölkerung verbessert, d.h. ökologische Zusammenhänge könnten erklärt und Hinweise auf einfache Schutzmaßnahmen (wie guardarayas, Erdwälle als Erosionsschutz, Polykulturen usw.) erteilt werden.

NATIONS (1981) meint voller Enthusiasmus: «By combining the environmental wisdom of the Lacandon Maya with the technological advances of Western agricultural research, we could create new systems of food and fiber production that are compatible with rainforest preservation and regeneration.»

\subsubsection{Hortikultur}

Eine Vielzahl von Zierpflanzen wird durch die Bevölkerung angebaut, und die Blumen werden z.T. auf den Märkten zum Verkauf angeboten. Mit wenig Aufwand ließen sich Araceaen, Bromelien oder Orchideen ziehen, ohne da $\beta$ es dabei zu Artengefährdung käme. Jeder größere Baum beherbergt beispielsweise 6-7 verschiedene Orchideenarten, die beim Fällen der Bäume natürlich vernichtet werden. Solche Pflanzen von gefällten Bäumen könnten gesammelt und auf neuen Wirtsbäumen festgebunden werden. Bestehende Baumplantagen in der Nähe von Flugpisten würden sich besonders dazu eignen. Die Schnittblumen könnten per Flugzeug über Palenque oder Tenosique nach den USA geflogen werden, wie dies bereits mit Shate (Chamaedorea sp.) geschieht.

\section{Literaturverzeichnis}

ACOPA, D. \& CALLEROS, G. (1981): Proyecto Forestál, Secretaria Forestál y de la Fauna, Palenque, Chiapas.

BRAUER, F. (1980): Comprar Maiz ò producirlo el mismo, Analisis Critico-Histórico de la Comunidad Lacandona, Programa Ecológico Selva Lacandona, Desarrollo Forestál, Palenque, Chiapas.

CETENAL (1975/76): Proyecto Selva Lacandona, México, D. F.

DUBY-BLOM, G. (1981): Jahresbericht-Rundschreiben an ihre Freunde und Gönner, San Cristobal.

FAO (1981): Los recursos forestales de la America tropical, proyecto de evaluación de los recursos forestales tropicales, FAO, Roma.

FRANKE, W. (1976): Nutzpflanzenkunde, nutzbare Gewächse der gemässigten Breiten, Subtropen und Tropen, Georg Thieme Verlag. Stuttgart.

GELBERT, M. (1982): Roza, Tumba y Quema, Shifting Cultivation und Waldveränderungen in der Selva Lacandona (Mexiko), Diplomarbeit (MS), Zürich.

MUENCH-NAVARRO, P.E. (1978): Los sistemas de producción agricola en la Región Lacandona, Estúdio agronómico preliminar, Tesis, Universidad Autónoma Chapingo, Chapingo, México.

NATIONS, J.D. (1981): The Rainforest Farmer, Pacific Discovery, February 1981.

\section{Resumen}

La agricultura semi-nómada de la Selva Lacandona tiene dos ciclos principales que se intercambian. El ciclo «milpa de año» empieza con la roza a principios de año y continua con el razgado de los árboles mayores, la tumba de estos, la quema, la siembra del maíz y los frijoles, la dobla del maíz y termina con la cosecha entre Septiembre y Octubre. En el ciclo «tornamil» no son necesarios ni la tumba ni la quema. Los frijoles se siembran a finales de Noviembre y el maíz a principios de Diciembre. La recogida es entre Mayo y Julio.

La agricultura semi-nómada es la actividad económica más importante de la Selva Lacandona, pero existe una tendencia progresiva hacia la ganadería. A causa de la gran emigración, ante todo de los habitantes de los Altos de Chiapas, la destrucción de los bosques húmedos tropicales avanza muy deprisa. De las 470000 hectareas de tierra, enpotencia, cultivada hay (1980) 253000 has. en cultivo y de 200000 a 250000 has. de vegetación secundaria en barbecho. Daños debidos a la erosión se ven en diversos sitios, sobre todo donde el bosque fue hace muchos años destruido. Esto sucede por ejemplo en la zona de entre los meandros del Usumacinta y los cerros.

SARUKHAN, K.J. \& PENNINGTON, T.D. (1968): Los àrboles tropicales de México, INIF-FAO, México.

SPENCER, J.E. (1966): Shifting Cultivation in Southeastern Asia, UCLA Publications in Geography, Vol.19, Berkeley and Los Angeles.

TURRENT-FERNANDEZ, C. (1978): Agroecosistema cultivos mixtos semipermanentes (roza - tumba - quema) en la Sierra de Tabasco, México, Tesis Ing. Agr., México.

WATTERS, R. F. (1971): La agricultura migratoria en la America Latina, FAO, Roma.

WEISCHET, W. (1980): Die ökologische Benachteiligung der Tropen, B. G. Teubner, Stuttgart.

\section{Anmerkungen:}

(1) Ejido: Geschlossene, abgegrenzte Landeinheit, oft um einen alten Siedlungskern. Kommunalland mit Individualund/oder Kommunalbesitz. Die bearbeitete Parzelle geht nach dem Tod des Vaters in den Besitz des ältesten Sohnes bzw. der Mutter über und darf nicht verkauft werden.

(2) Genauere Zahlenangaben sind jedoch nicht erhältlich. 